\title{
Conventional Endodontic Retreatment of Persistent Pain on Previously Treated Tooth in an Elderly Patient: A Case Report
}

\author{
Nina Dhaniar, DDS, ${ }^{1}$ Hermawan Adi Praja, DDS, ${ }^{1}$ Ratih Mahanani Santoso, DDS, ${ }^{1}$ \\ Cendranata Wibawa Ongkowijoyo, DDS, MDPPH ${ }^{1}$ and Widya Saraswati, DDS, MDSC, $\mathrm{PhD}^{2}$ \\ ${ }^{1}$ Student of Specialist Program of Conservative Dentistry Department, Faculty of Dental Medicine, Universitas Airlangga \\ ${ }^{2}$ Lecturer of Specialist Program of Conservative Dentistry Department, Faculty of Dental Medicine, Universitas Airlangga
}

\begin{abstract}
Clinical evaluation for a successful root canal treatment is assessed by various criteria, which are clinical, histopathological, and radiographical criteria. Therefore, failure of endodontic treatment can be described as a recurrence of clinical symptoms, with the presence of a periapical radiolucency or both. Failure factors in the treatment are frequently related to persistent infection. Conventional endodontic retreatment is indicated for symptomatic previously treated teeth or asymptomatic teeth with inadequately done initial endodontic treatment to avoid potential recurrence. Endodontic retreatment in elderly patients is a great challenge because the clinician has to reassure both the physical and psychological factors of the patient to determine whether to save a tooth or perform an extraction. Some difficulties may also be found in root canal retreatment, including finding the root canal hole or root canal blockage found in parts of the root canal that have not been repaired in the previous treatment.
\end{abstract}

A 60-year-old female patient came with the chief complaint of recurrent pain, and subjective discomfort in the maxillary left central incisor. The patient had anxiety about the dental treatment. The tooth had a history of root canal treatment four months ago.

The clinical examination showed a positive response to the percussion test. The radiographical analysis showed a root canal underfilling, 2-3 mm short of length from the apex. The tooth was diagnosed as a previously treated tooth with symptomatic apical periodontitis. Endodontic retreatment was performed based on the patient's clinical condition and consent, followed by composite restoration.

The clinical and radiographic re-evaluation after four weeks of follow-up revealed an excellent condition. This favorable result showed that a conventional retreatment plan of persistent pain on the previously treated tooth in an elderly patient led to progressive healing, and a longer follow-up was advised.

Key Words: elderly patient, endodontic retreatment, persistent pain, previously treated tooth

\section{INTRODUCTION}

Paper presented in the Temu Ilmiah Nasional-Internasional ke-8 (TIMNASS 8) on February 27-29, 2020 at Surabaya, Indonesia.

Corresponding author: Widya Saraswati, DDS, MDS, PhD Lecturer of Specialist Program of Conservative Dentistry Department

Faculty of Dental Medicine

Universitas Airlangga

Jl. Airlangga No.4 - 6, Airlangga, Kec. Gubeng,

Kota SBY, Jawa Timur 60115, Indonesia

Email: widya.fkgunair@gmail.com
Studies of the success and failures of conventional endodontic treatments have been done. An adequate treatment has been presented in $95 \%$ of the cases. Along with that success rate comes the unavoidable percentage of unsuccessful treatments.

Clinical evaluation for success root canal treatment is assessed by various criteria, which are clinical, histopathological, and radiographical. Although the failure of endodontic treatment is not yet apparent, it can be described as a recurrence of clinical symptoms, the presence of a periapical radiolucency, or both. The clinical criteria include 
no pain on percussion or palpation, no tooth mobility, no subjective discomfort, normal function and esthetics, no swelling sign, no sinus tract, no periodontal disease, and minimal to no occurrence of discoloration. ${ }^{1,2}$ Failure factors in the treatment are frequently related to persistent infection due to inadequate cleaning, shaping, and filling of the root canal system. It can be directly and indirectly associated with bacteria inside the root canal. ${ }^{2}$ Unsuccessful root canal treatment can be caused by biological failing, including no rubber dam, incorrect irrigants, inability to prepare along the length of the canal, missed canals, and inadequate obturation. It also can be caused by root fracture, inadequate restoration, and resistant microorganisms. ${ }^{3}$

Conventional endodontic retreatment is one of the options available for non-healing root canal-treated teeth. A non-healing tooth is considered restorable where the patient is motivated to preserve her natural dentition, periodontally sound, and further endodontic treatment is reasonable. Retreatment should be the first option to consider. The patient has to understand the time-consuming procedure, the risks, the benefits, and the retreatment cost. Retreatment procedure aims to give the clinician the chance to eradicate microorganisms that have been left behind during the previous treatment and those that may have penetrated the root canal after the last treatment. ${ }^{4}$

Elderly patients highly appreciate conventional endodontic treatment due to changes in priorities. The difference from those of younger patients may influence the treatment plan. Elderly patients are more intently driven by short-term objectives and asymptomatic function than by aesthetics and long-time goals in treatment. Conventional endodontic treatment is highly valued because of pain relief, chewing and digestion adjustment, aesthetic and self-esteem improvement, and improved preservation of natural dentition. However, there are negative sentiments concerning the endodontic treatment in some elderly patients, including prolonged dental visits, prolonged time that the mouth must stay open, dryness of the mouth, discomfort from rubber dam isolation, high cost, fear of pain and limited flexibility. ${ }^{5}$ Patients with anxiety about dental treatment require special care in identifying and reducing the negative impacts for better results.

The following case report aims to present a case where conventional endodontic retreatment was performed in a previously treated maxillary left central incisor with persistent pain in an elderly patient with anxiety.

\section{CASE REPORT}

A 60-year-old female patient presented with a chief complaint of recurrent pain and subjective discomfort on chewing in the maxillary left central incisor. The tooth had a history of root canal treatment by a general practitioner four months ago due to caries and severe throbbing pain. The pain intensity was decreasing, but it did not heal completely after the treatment. The clinical examination showed a temporary filling (Figure 1A) and a positive response to the percussion test. The radiographical analysis showed a $3 \mathrm{~mm}$ root canal underfilling and periodontal ligament thickening (Figure 1B). The inadequate root canal filling was considered as the reason for failure. The tooth was diagnosed as a previously treated tooth with symptomatic apical periodontitis. The patient said she had a fear of dental treatment. The patient had no medical compromises and denied any major systemic diseases.

\section{CASE MANAGEMENT}

The treatment of choices given to the patient included conventional endodontic retreatment or extraction and prosthetic replacement. The procedures were described and explained, including the risks and benefits. The patient opted to preserve her natural dentition and minimally invasive therapy. The clinician decided to do endodontic retreatment followed by composite restoration after the clinical examination, taking the patient's preference (consent) and the possibility of cooperation associated with the anxiety.
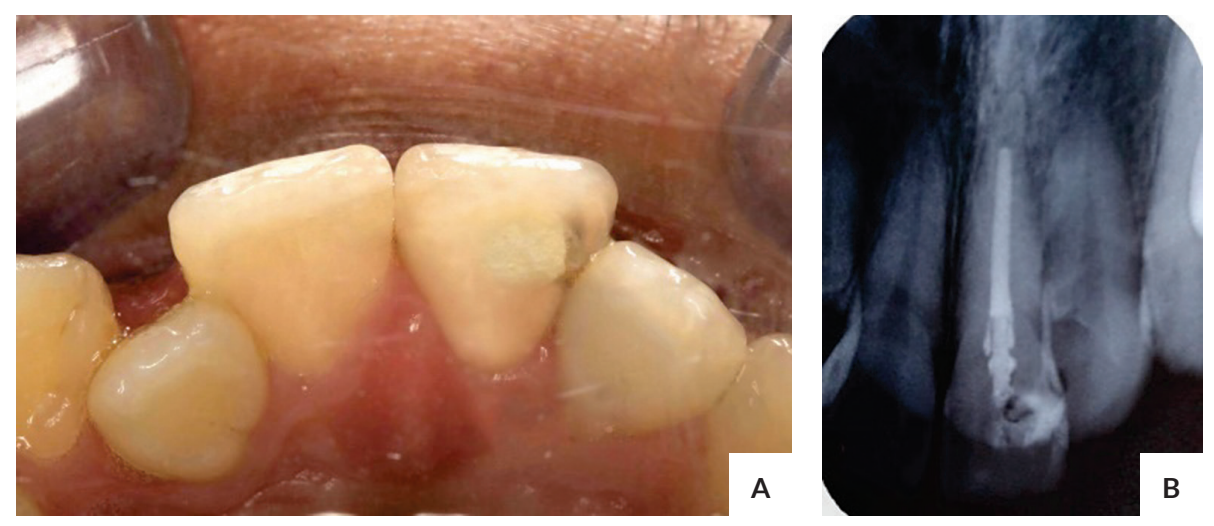

Figure 1. (A) Pre-operative clinical appearance; (B) Pre-operative periapical radiograph. 

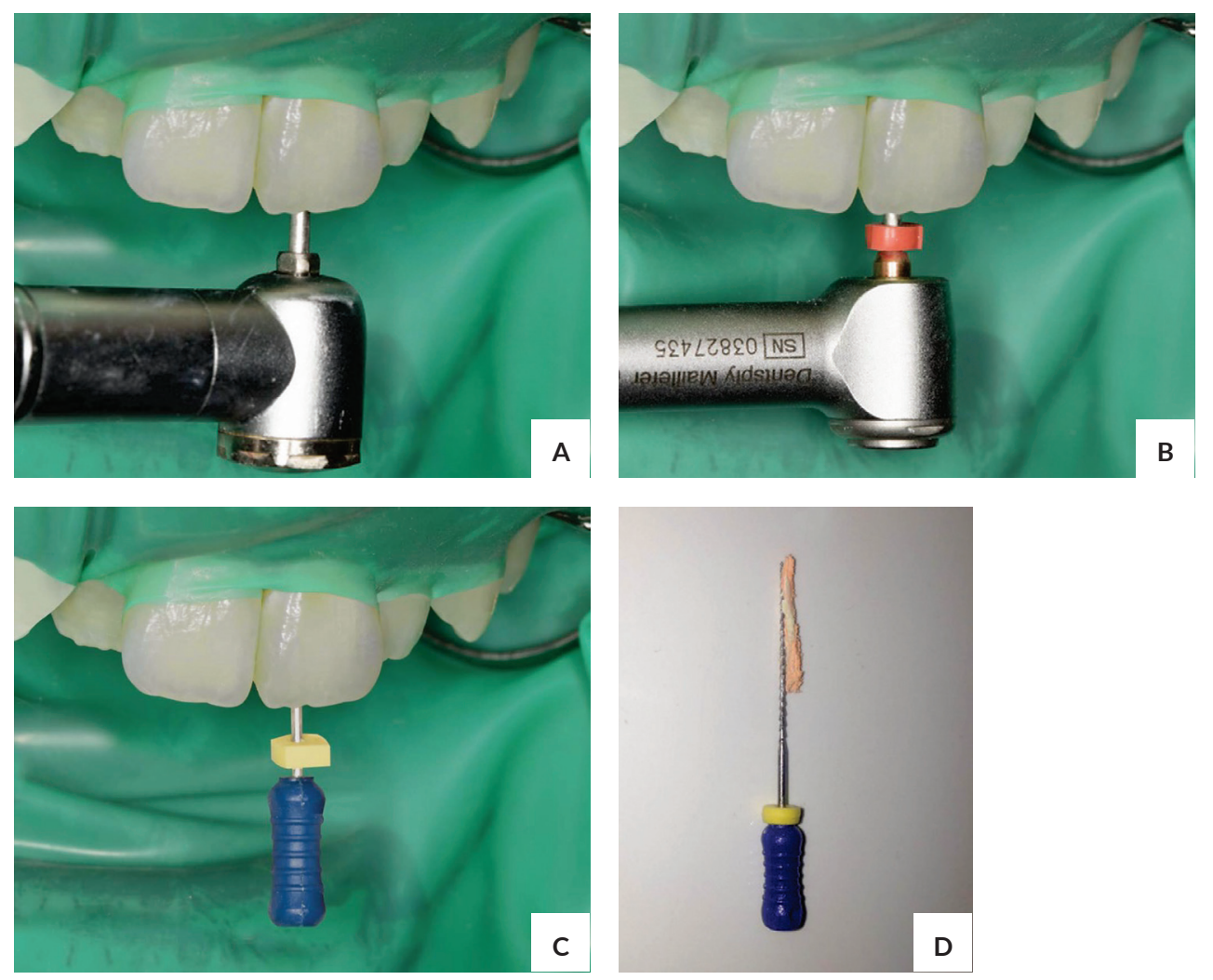

Figure 2. (A) Access Opening; (B) Coronal half gutta-percha removal using Reciproc R25; (C) Apical half gutta-percha removal using $\mathrm{H}$-file; (D) The $\mathrm{H}$-file removed gutta-percha.

A rubber dam was applied using the anterior quadrant isolation technique; caries was removed with a round diamond bur, and the root canal was accessed (Figure 2A). A pilot hole was made on the gutta-percha using a heated plugger. The coronal half of the gutta-percha was removed with a reciprocating motion instrument Reciproc R25 (RECIPROC ${ }^{\circledR}$ blue, VDW, Munich, Germany) (Figure 2B). In contrast, the apical part was removed with the Hedstroem file (H-file \#30 Dentsply, Maillefer, Ballaigues, Switzerland) (Figure 2C-D). A periapical radiograph was performed to confirm the gutta-percha removal.

Negotiation, glide path, and patency was done using a K-file (25mm K-file \#20 Dentsply, Maillefer, Ballaigues, Switzerland). Apex Locator (Root ZX, J. Morita Corp, Tokyo, Japan) was used to determine the working length. The working distance was $23 \mathrm{~mm}$ and was confirmed by the periapical radiograph (Figure 3A). Root canal shaping was performed using ProTaper Next X1-X4 (PTN, Dentsply, Maillefer, Ballaigues, Switzerland), and cleaning was done using 17\% EDTA, 2.5\% $\mathrm{NaOCl}$, and Aquadest (Figure 3B-C). The root canal was sterilized using Calcium Hydroxide paste (Figure 3D), and the cavity was sealed using Cavit temporary filling. The patient was scheduled for the next appointment in one week.
On the second appointment, the tooth was asymptomatic. The temporary filling remained good. The tooth was accessed after the isolation was installed. Activated irrigation was performed using EDTA 17\%, Aquadest, $2.5 \% \mathrm{NaOCl}, 30 \mathrm{G}$ side vented irrigation needle, and Endoactivator (EndoActivator ${ }^{\circledR}$, Dentsply, Tulsa Dental Specialities, Tulsa, OK, USA) (Figure 4A). The tooth was dried using paper points and obturated using GuttaPercha ProTaper Next X4 and AH Plus sealer (Figure 4B). The tooth was then restored with composite resin (Filtek Z350XT shade A3, 3M ESPE, USA), and a postoperative periapical radiograph was done (Figure $4 \mathrm{C}-\mathrm{D}$ ).

Clinical and re-evaluation after four weeks followup revealed no pain and subjective discomfort reported an excellent condition of the restoration and the periapical area of the tooth (Figure 4E).

\section{DISCUSSION}

The success rate of retreatment was considered to be lower than the previous root canal treatment. Retreatment could have a similar result with the last root canal treatment if no canal blocks sealed the access to the apex. 

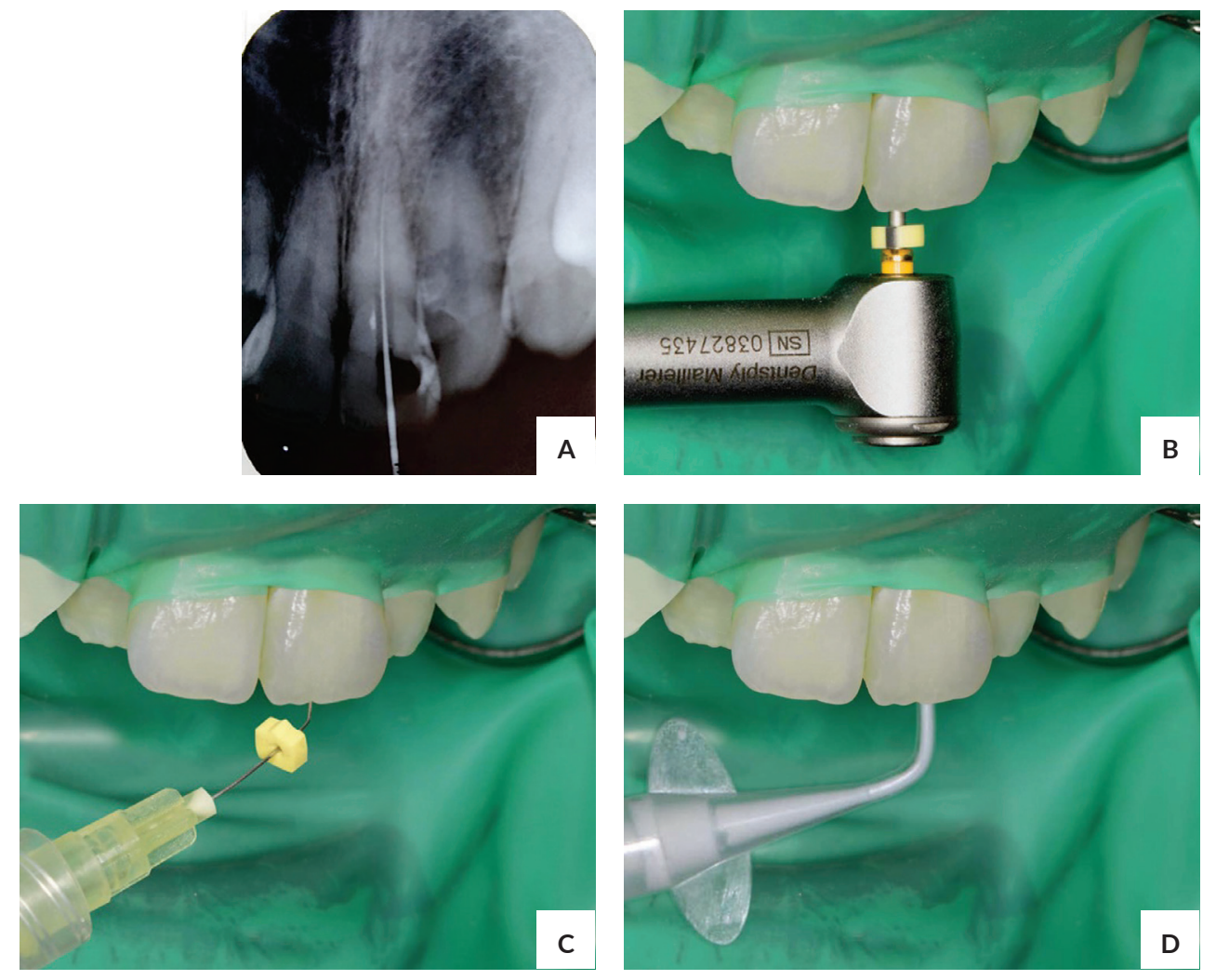

Figure 3. (A) Working Length radiograph confirmation; (B) Shaping; (C) Cleaning; (D) Dressing.
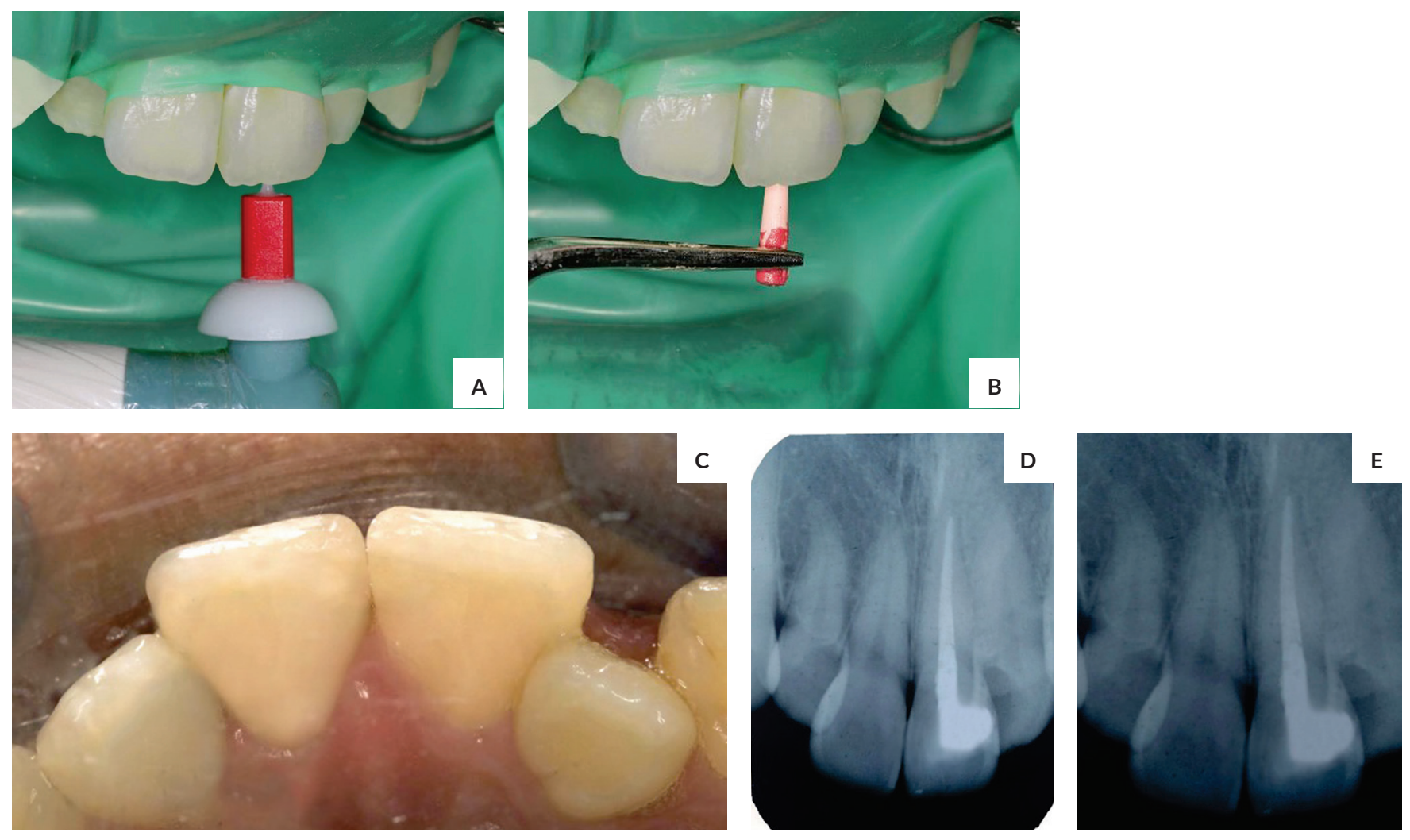

Figure 4. (A) Activated irrigation; (B) Obturation; (C) Final restoration; (D) Post-operative periapical radiograph; (E) Periapical radiograph of 4 weeks follow up. 
Conventional root canal retreatment has been reported successful in $74-75 \%$ of cases. Factors that should be considered in determining the prognosis of the retreatment are periapical lesions, the condition of previous treatment, the condition of coronal restoration, and the existence of iatrogenic factors. ${ }^{6,7}$

Retreatment procedure would be the treatment of choice after several considerations that make it feasible: the tooth is restorable, periodontally sound, and the root canal accessible nonsurgical. The clinicians have to do a thorough examination, utilizing available technology to determine a treatment plan.

In the present case, a retreatment decision was made based on poor obturation and persistent pain. Although endodontic surgery offers more favorable short-term success rates, nonsurgical root canal retreatment provides more favorable long-term results. There may be microorganisms left in the root canal because of inadequate obturation done in the previous treatment, or new ones may come from microleakage. The microorganisms trigger the immune system to release inflammation mediators as a defense mechanism. The inflammation mediators can cause the thickening of the periodontal ligament or the periapical lesion. ${ }^{4,8}$

The instruments used in this case, Reciproc R25 and Hedstroem file, effectively removed the gutta-percha. Previous studies showed that retreatment instruments could not clean the canal space thoroughly from the filling materials. Therefore, the irrigation procedure was needed to optimize cleaning of the residual filling materials. ${ }^{9}$

After the periapical radiograph was performed to ensure the gutta-percha removal, the K-file was used to negotiate, confirm the canal's path, and provide the canal entrance for preparation. Determining the working length is an essential step in endodontic retreatment. A periapical radiograph was used to confirm the score from the apex locator. Root canal preparation was performed using the NiTi instrument, ProTaper Next X1-X4, because it was good to produce a bigger tapper for obturation.

The crown down technique of ProTaper is a technique that prepares the coronal of the canal before the apical part. The flaring in the coronal part will ease the apical part preparation. The cleaning and shaping in the crown down technique will minimize the risk of irritant extrusion in the periapical tissue.

The cleaning was done using 17\% EDTA, 2.5\% NaOCl, and Aquadest. To dissolve the smear layer, 17\% EDTA was used. Inorganic debris can decrease the ability of the sealer to penetrate the dentin tubules, while $2.5 \% \mathrm{NaOCl}$ was used as an anti-microbial agent. Both EDTA and $\mathrm{NaOCl}$ are effective cleaning irrigants if used in combination. ${ }^{8}$

The root canal was sterilized using calcium hydroxide paste to eliminate microorganisms and to avoid recurrence. Calcium hydroxide starts the mineralization and boosts the metabolic process of the periapical tissue. ${ }^{10}$ The sealer used was resin-based for its excellent sealing ability. The final restoration, in this case, was composite resin. It was chosen because it is a superb treatment option as a minimally invasive restoration for small cavities treated endodontic teeth.

Elderly patients highly appreciate conventional endodontic treatment because of pain relief, chewing and digestion adjustment, and improved preservation of the natural dentition. However, there are negative sentiments concerning endodontic therapy in some elderly patients, including prolonged dental visits, prolonged time that the mouth must stay open, dryness of the mouth, discomfort from rubber dam isolation, high cost, fear of pain, and limited flexibility. Therefore, there should be a consideration that clinicians have to understand in treating elderly patients.

Informed consent is essential, especially for medically compromised elderly patients. Medical history, current medications, emotional needs, physical needs, and treatment time are other factors considered. ${ }^{5}$

Some difficulties may be found in root canal retreatment, including finding the root canal hole or root canal blockage found in parts of the root canal that have not been repaired in the previous treatment. This is due to the shrinkage caused by the aging of the pulp chamber in the crown and root canal walls. In addition, it is necessary to pay attention to the working length of the teeth in the elderly group because of aging. The working distance can be reduced from the average length of normal teeth. This occurs due to attrition and cement at the root tip of a physiological process or interferes with resorption. ${ }^{5}$

Dental fear is a reaction to stimuli that threaten the tooth. ${ }^{11}$ Phobia is a constant, unrealistic, and intense fear of specific stimuli, which leads to avoidance of perceived danger. An extraordinary and irrational fear of dentistry related to fear due to hypertension, terror, and anxiety is called "odontophobia".12 Odontophobia can harm a person's quality of life. Therefore, it is crucial to identify and reduce these significant obstacles to open the way for better oral health. It is the duty and responsibility of the dentist to give excellent dental care to these patients with special needs. The management of these patients must be an integral part of clinical practice because a large portion of the population suffers from anxiety and fear.

After proper evaluation, therapy must be adjusted based on experience, expertise, dentist anxiety level, patient intelligence, age, cooperation, and clinical situation. The dentist must communicate with the patient and identify the source of their fear and anxiety. In addition, use selfreported anxiety and fear scales and allow categorization as mild, moderate, or extreme anxiety or dental phobia. Various psychological therapies relieve emotional, cognitive, behavioral, and physiological dimensions of dental anxiety and fear. Communicating the treatment schedule with treatment sessions that are not too long. is very important in the treatment plan for patients with high fear. ${ }^{13}$ The main focus of the dental team should be efficient long-term 
therapy with positive effects on patients, which will enable them to seek dental care in the future.

Patients with mild and moderate anxiety can often be managed using psychological intervention and sometimes anxiolytic medication or conscious sedation. Patients who are very anxious or phobic most often require a combined management approach. Because of the high risks involved in pharmacological interventions, dentists and the dentist team must follow appropriate guidelines. They should be adequately trained and equipped with enough infrastructure before pharmacological interventions can be included. All successful treatments rely on dentist-patient collaboration. Thus, a relaxed patient will produce a less stressful atmosphere for the dentist, and a better treatment outcome. ${ }^{14}$ Hopefully, this case report will help other clinicians know how to deal with the elderly in endodontic retreatment.

\section{CONCLUSION}

The clinicians have to do thorough examinations, utilizing available technology to determine the treatment plan of previously treated teeth. Clinicians have to consider several factors in treating elderly patients. The favorable result in this case report showed that a conventional retreatment plan with persistent pain on a previously treated tooth in an elderly patient led to progressive healing, although a longer follow-up was advisable.

\section{Statement of Authorship}

All authors participated in the data collection and analysis and approved the final version submitted.

\section{Author Disclosure}

All authors declared no conflicts of interest.

\section{Funding Source}

This study was funded by the Universitas Airlanggga.

\section{REFERENCES}

1. Ingle J, Bakland L, Baumgartner J. Ingle's Endodontics 7. Hamilton: BC Decker Inc; 2019. pp. 729-35.

2. Garg N, Garg A, Nekoofar MH. Textbook of Endodontics. 4th ed. New Delhi: Jaypee Brothers Medical Publishers; 2014. pp. 345-9.

3. Ford TR, Rhodes JS. Root Canal Retreatment: I. Case Assessment and Treatment Planning. Dent Update. 2004 Jan-Feb;31(1):34-9. doi:10.12968/denu.2004.31.1.34. PMID: 15000007.

4. Torabinejad M, Walton RE, Fouad AF. Endodontic Principles and Practice. 5th ed. St. Louis, Missouri: Elsevier; 2015. pp. 352-61.

5. AlRahabi MK. Root Canal Treatment in Elderly Patients: A Review and Clinical Considerations. Saudi Med J. 2019 Mar;40(3):21723. doi: 10.15537/smj.2019.3.23769. PMID: 30834415; PMCID: PMC6468204.

6. Economides N, Vouzara T. Nonsurgical Endodontic Retreatment after Unsuccessful Apicectomy: A Case Report. Balk J Stom. 2011;15(3):166-70.

7. Torabinejad M, White SN. Endodontic Treatment Options after Unsuccessful Initial Root Canal Treatment: Alternatives to Singletooth Implants. J Am Dent Assoc. 2016 Mar;147(3):214-20. doi: 10.1016/j.adaj.2015.11.017. Epub 2016 Jan 9. PMID: 26778004.

8. Wijaya HY, Karlina S. Nonsurgical Endodontik Retreatment of Maxillary First Premolar: A Case Report. Denta. 2017 Feb;11(1): 88-95.

9. De-Deus G, Belladonna FG, Zuolo AS, Simões-Carvalho M, Santos CB, Oliveira DS, Cavalcante DM, Silva EJNL. Effectiveness of Reciproc Blue in Removing Canal Filling Material and Regaining Apical Patency. Int Endod J. 2019 Feb;52(2):250-257. doi: 10.1111/ iej.12991. Epub 2018 Sep 8. PMID: 30091141.

10. Dewiyani S. Calcium Hydroxide as Intracanal Dressing for Teeth with Apical Periodontitis. Dental Journal (Majalah Kedokteran Gigi). 2011;46(1):12-6.

11. Caltabiano ML, Croker F, Page L, Sklavos A, Spiteri J, Hanrahan L, Choi R. Dental Anxiety in Patients Attending a Student Dental Clinic. BMC Oral Health. 2018 Mar 20;18(1):48. doi: 10.1186/s12903-0180507-5. PMID: 29558935; PMCID: PMC5859659.

12. De Stefano R. Psychological Factors in Dental Patient Care: Odontophobia. Medicina (Kaunas). 2019 Oct 8;55(10):678. doi: 10.3390/medicina55100678. PMID: 31597328; PMCID: PMC6843210.

13. Appukuttan DP. Strategies to Manage Patients with Dental Anxiety and Dental Phobia: Literature Review. Clin Cosmet Investig Dent. 2016 Mar 10;8:35. doi: 10.2147/CCIDE.S63626. PMID: 27022303; PMCID: PMC4790493.

14. Sumidart Ai, Dwiandhany WS. Management Pain and Anxiety in Endodontic Treatment. J Dentomaxillofac Sci. 2017;2:4-6.

\section{The Acta Medica Philippina is now accepting limited advertising for its front and back cover (colored), as well as for available spaces in some of its pages, as appropriate. For inquiries and submission of proposals, please email us at actamedicaphilippina.upm@up.edu.ph}

